\title{
Risk-Sensitive Asset Management under a Wishart Autoregressive Factor Model
}

\author{
Hiroaki Hata ${ }^{1}$, Jun Sekine ${ }^{2}$ \\ ${ }^{1}$ Department of Mathematics, Faculty of Education, Shizuoka University, Ohya, Japan \\ ${ }^{2}$ Division of Applied Mathematics for Social Systems, Graduate School of Engineering Science, Osaka University, \\ Toyonaka, Japan \\ Email: ehhata@ipc.shizuoka.ac.jp, sekine@sigmath.es.osaka-u.ac.jp
}

Received December 25, 2012; revised January 28, 2013; accepted February 15, 2013

\begin{abstract}
The risk-sensitive asset management problem with a finite horizon is studied under a financial market model having a Wishart autoregressive stochastic factor, which is positive-definite symmetric matrix-valued. This financial market model has the following interesting features: 1) it describes the stochasticity of the market covariance structure, interest rates, and the risk premium of the risky assets; and 2) it admits the explicit representations of the solution to the risk-sensitive asset management problem.
\end{abstract}

Keywords: Risk-Sensitive Asset Management; Wishart Autoregressive Stochastic Factor; Stochastic Covariance; Stochastic Interest Rate; Stochastic Risk Premium; Riccati Differential Equation

\section{Introduction}

\subsection{Risk-Sensitive Asset Management}

Consider a continuous-time financial market that consists of one riskless asset and $n$ risky assets. The price process of the riskless asset $S^{0}:=\left(S_{t}^{0}\right)_{t \geq 0}$ and that of the $n$ risky assets $S:=\left(S^{1}, \cdots, S^{n}\right)^{\mathrm{T}}, S^{i}:=\left(S_{t}^{i}\right)_{t \geq 0}$, where $(\cdot)^{\mathrm{T}}$ denotes the transpose of a vector or matrix, are semimartingales defined on a filtered probability space $\left(\Omega, \mathcal{F}, \mathbb{P},\left(\mathcal{F}_{t}\right)_{t \geq 0}\right)$. Define the wealth process $X^{\pi}:=\left(X_{t}^{\pi}\right)_{t \geq 0}$ of a self-financing investor governed by the following stochastic differential equation (SDE):

$$
\begin{aligned}
& \frac{\mathrm{d} X_{t}^{\pi}}{X_{t}^{\pi}}=\left\{\sum_{i=1}^{n} \pi_{t}^{i} \frac{\mathrm{d} S_{t}^{i}}{S_{t}^{i}}+\left(1-\sum_{i=1}^{n} \pi_{t}^{i}\right) \frac{\mathrm{d} S_{t}^{0}}{S_{t}^{0}}\right\}, \\
& X_{0}^{\pi}=x,
\end{aligned}
$$

where $x \in \mathbb{R}_{++}$is the initial wealth of the investor, and $\pi:=\left(\pi^{1}, \cdots, \pi^{n}\right)^{\mathrm{T}}, \pi^{i}:=\left(\pi_{t}^{i}\right)_{t \geq 0}$ is the dynamic investment strategy of the investor. Let

$$
G_{t}^{\pi}:=\frac{1}{t} \log X_{t}^{\pi},
$$

be the growth rate of the wealth at time $t$. For given constants $T \in(0, \infty)$ and $\gamma \in(0, \infty)$, define the risksensitized expected value of $G_{T}^{\pi}$ by

$$
C_{T}^{\pi}:=\frac{1}{(-\gamma T)} \log \mathbb{E} \exp \left(-\gamma T G_{T}^{\pi}\right),
$$

which is rewritten as

$$
\exp \left(-\gamma T G_{T}^{\pi}\right)=\mathbb{E} \exp \left(-\gamma T G_{T}^{\pi}\right)
$$

and interpreted as the certainty equivalent value of $G_{T}^{\pi}$ with respect to the exponential criterion function $\exp (-\gamma T(\cdot))$. We are interested in maximizing $G_{T}^{\pi}$, that is,

$$
\begin{aligned}
\Gamma_{T}(\gamma) & :=\sup _{\pi \in \cdot_{T}} \frac{1}{(-\gamma T)} \log \mathbb{E} \exp \left(-\gamma T G_{T}^{\pi}\right) \\
& =\sup _{\pi \in \sim_{T}} \frac{1}{(-\gamma T)} \log \mathbb{E}\left(X_{T}^{\pi}\right)^{-\gamma},
\end{aligned}
$$

which we call the risk-sensitive asset management problem. Here, $\mathscr{A}_{T}$ is a space of admissible investment strategies and is a subset of $\mathscr{L}_{2, T}^{n}$, the totality of $n$-dimensional $\mathcal{F}_{t}$-progressively measurable processes $\left(p_{t}\right)_{t \in[0, T]}$ on the time interval $[0, T]$ such that $\int_{0}^{T}\left|p_{t}\right|^{2} \mathrm{~d} t<\infty$ almost surely.

Remark 1.1 The risk-sensitive asset management problem (1.3) has been well-studied under a linear-Gaussian market model, for example, by [1-7]. In those works, the price processes $\left(S^{0}, S\right)$ are given by the solutions to the following system of SDEs:

$$
\begin{aligned}
& \mathrm{d} S_{t}^{0}=S_{t}^{0} r\left(Y_{t}\right) \mathrm{d} t, \quad S_{0}^{0}=1, \\
& \mathrm{~d} S_{t}=\operatorname{diag}\left(S_{t}\right)\left\{\mu\left(Y_{t}\right) \mathrm{d} t+\Sigma \mathrm{d} W_{t}\right\}, \quad S_{0} \in \mathbb{R}_{++}^{n}, \\
& \mathrm{~d} Y_{t}=b\left(Y_{t}\right) \mathrm{d} t+\Lambda \mathrm{d} W_{t}, \quad Y_{0} \in \mathbb{R}^{m}
\end{aligned}
$$


on a filtered probability space $\left(\Omega, \mathcal{F}, \mathbb{P},\left(\mathcal{F}_{t}\right)_{t \geq 0}\right)$ endowed with the $d$-dimensional $\mathcal{F}_{t}$-Brownian motion $W:=\left(W^{1}, \cdots, W^{d}\right)^{\mathrm{T}}, W^{i}:=\left(W_{t}^{i}\right)_{t \geq 0}$. Here, $\operatorname{diag}(x)$ denotes the diagonal matrix whose $(i, i)$ th element is equal to the $i$ th element $x^{i}$ of $x:=\left(x^{1}, \cdots, x^{n}\right)^{\mathrm{T}} \in \mathbb{R}^{n}$, $\Sigma \in \mathbb{R}^{n \times d}, \Lambda \in \mathbb{R}^{m \times d}$, and

$$
\begin{aligned}
& r(y):=r_{0}+r_{i}^{\mathrm{T}} y, \\
& \mu(y):=r(y) \mathbf{1}+\theta(y), \\
& \theta(y):=\theta_{0}+\Theta_{1} y, \\
& b(y):=b_{0}+B_{1} y
\end{aligned}
$$

with $\quad r_{0} \in \mathbb{R}, \quad r_{1} \in \mathbb{R}^{m}, \quad \mathbf{1}:=(1, \cdots, 1)^{\mathrm{T}} \in \mathbb{R}^{n}, \quad \theta_{0} \in \mathbb{R}^{n}$, $\Theta_{1} \in \mathbb{R}^{n \times m}, \quad b_{0} \in \mathbb{R}^{m}$, and $B_{1} \in \mathbb{R}^{m \times m}$. We reformulate (1.3) with (1.1), (1.2), (1.4), and (1.5) as a linear exponential quadratic Gaussian stochastic control problem, and the optimal investment strategy (portfolio)

$\hat{\pi}^{(T)}:=\left(\hat{\pi}_{t}^{(T)}\right)_{t \in[0, T]}$ for (1.3) is represented explicitly:

$\hat{\pi}_{t}^{(T)}:=\frac{1}{1+\gamma}\left(\Sigma \Sigma^{\mathrm{T}}\right)^{-1} \theta\left(Y_{t}\right)-\frac{\gamma}{1+\gamma}\left(\Sigma \Sigma^{\mathrm{T}}\right)^{-1} \Sigma \Lambda^{\mathrm{T}}\left(p_{t}+P_{t} Y_{t}\right)$.

Here, $\left(P_{t}\right)_{t \in[0, T]}$ is the solution to a matrix differential Riccati equation, and $\left(P_{t}\right)_{t \in[0, T]}$ is the solution to a linear differential equation, including $P$.

Remark 1.2 Intuitively, recalling the cumulant expansion,

$$
C_{T}^{\pi}=\mathbb{E} G_{T}^{\pi}-\frac{\gamma}{2} \mathbb{V} \sqrt{T} G_{T}^{\pi}+O\left(\gamma^{2}\right) \quad \text { as } \gamma \rightarrow 0,
$$

where $\mathbb{V}[\cdot]$ denotes variance, we interpret (1.3) as a risk-sensitized optimization of the expected growth rate maximization,

$$
\sup _{\pi} \mathbb{E} G_{T}^{\pi}
$$

\subsection{Wishart Factor Model}

The main aim of the present paper is to introduce a simple and tractable market model that satisfies the following requirements:

- The model describes the stochasticity of the covariance structure of $\left(\frac{\mathrm{d} S^{i}}{S^{i}}, \mathrm{~d} Y\right)(i=1, \cdots, n)$, interest rates, and mean-return rates of $S$.

- The model admits an explicit representation of the optimal investment strategy for (1.3).

For the purpose, we employ a Wishart autoregressive process as a stochastic factor, which is positive-definite symmetric matrix-valued. Such matrix-valued processes have been introduced and studied by [8], and recently, generalizations have been intensively studied, for example, see $[9,10]$, and the references therein. Moreover, these processes are now extensively utilized for financial modeling. We can refer to the examples given below.

- Modeling of multivariate stochastic volatility (covariance) under the risk-neutral probability: see [11-16].

- Modeling of multivariate asset price process under physical probability with stochastic covariance and mean-return rates: see $[14,17,18]$.

- Modeling of (term structure of) interest rates and stochastic intensity for credit risk: see [14,17,19,20].

Our market model defined by (2.1)-(2.4) in Section 2 is an extension of the model employed by [18], (see Example 2.1), who studied the expected CRRA-utility ma ximization of terminal wealth, which is essentially equivalent to (1.3). A main contribution of the present paper is a rigorous mathematical analysis of portfolio optimization problem (1.3) under a flexible Wishart autoregressive stochastic factor model: We strengthen the mathematical results in [18] by formulating an appropriate space of admissible trading strategies (see (3.5)) and showing a verification theorem for the candidate of the optimal strategy (see Theorem 3.1), both of which are omitted in [18].

In the next section, we introduce our market model with a Wishart autoregressive factor and present preliminary calculations of the associated Hamilton-JacobiBellman (HJB) equation for solving risk-sensitive asset management problem (1.3). In Section 3, we introduce our main results. In Section 4, we show the proof of the main theorem after preparing lemmas.

\section{Setup}

\subsection{Market Model with Wishart Autoregressive Factor}

Let $\left(\Omega, \mathcal{F}, \mathbb{P},\left(\mathcal{F}_{t}\right)_{t \geq 0}\right)$ be a filtered probability space endowed with the $(d \times d)$-dimensional $\mathcal{F}_{t}$-Brownian motion $B:=\left(B_{t}\right)_{t \in[0, T]}, \quad B_{t}:=\left(B_{t}^{i j}\right)_{1 \leq i, j \leq d}$, and the $d$-dimensional $\mathcal{F}_{t}$-Brownian motion $z:=\left(z_{t}\right)_{t \in[0, T]}$, $z_{t}:=\left(z_{t}^{1}, \cdots, z_{t}^{d}\right)^{\mathrm{T}}$, which is independent of $B$. Using a constant vector $\rho:=\left(\rho^{1}, \cdots, \rho^{d}\right)^{\mathrm{T}} \in \mathbb{R}^{d}$ so that $|\rho| \leq 1$, we define another $d$-dimensional Brownian motion

$$
\begin{array}{r}
w:=\left(w^{1}, \cdots, w^{d}\right)^{\mathrm{T}}, \quad w^{i}:=\left(w_{t}^{i}\right)_{t \geq 0} \text { by } \\
w_{t}:=B_{t} \rho+\sqrt{1-|\rho|^{2}} z_{t},
\end{array}
$$

which is correlated with $B$ as

$$
\mathrm{d}\left\langle w^{i}, B^{j k}\right\rangle_{t}=\delta_{i j} \rho^{k} \mathrm{~d} t
$$

where $\langle\cdot, \cdot\rangle$ denotes the quadratic covariation, and $\delta_{i j}$ denotes Kronecker's delta. We consider the price processes $\left(S^{0}, S\right)$, described by the following system of 
SDEs:

$$
\begin{aligned}
& \mathrm{d} S_{t}^{0}=r\left(Y_{t}\right) S_{t}^{0} \mathrm{~d} t, \\
& \mathrm{~d} S_{t}=\operatorname{diag}\left(S_{t}\right)\left\{\mu\left(Y_{t}\right) \mathrm{d} t+\Sigma \sqrt{Y_{t}} \mathrm{~d} w_{t}\right\}, \\
& \mathrm{d} Y_{t}=\left(L L^{\mathrm{T}}+K Y_{t}+Y_{t} K^{\mathrm{T}}\right) \mathrm{d} t+\sqrt{Y_{t}} \mathrm{~d} B_{t} \Lambda^{\mathrm{T}}+\Lambda \mathrm{d} B_{t}^{\mathrm{T}} \sqrt{Y_{t},}
\end{aligned}
$$

with the initial values $S_{0}^{0}=1, S_{0} \in \mathbb{R}_{++}^{n}$ and $Y_{0} \in \mathbb{S}_{++}^{d}$. Here, we denote by $\mathbb{S}^{d}$ the totality of $d$-dimensional, real, symmetric matrices, and $\mathbb{S}_{++}^{d}:=\left\{M \in \mathbb{S}^{d} ; M>0\right\}$. Furthermore, for $y \in \mathbb{S}^{d}$, we define

$$
\begin{aligned}
& r(y):=r_{0}+\operatorname{tr}\left(R_{1} y\right), \\
& \mu(y):=r(y) \mathbf{1}+\left(\Sigma y \Sigma^{\mathrm{T}}\right) \lambda,
\end{aligned}
$$

where $r_{0} \in \mathbb{R}, R_{1} \in \mathbb{S}^{d}$, and $\lambda \in \mathbb{R}^{n}$. Also, we assume that $\Sigma \in \mathbb{R}^{n \times d}$ is full rank, that is,

$$
\Sigma^{\mathrm{T}} \Sigma>0 \text { if } d \leq n \text {, and } \Sigma \Sigma^{\mathrm{T}}>0 \text { if } d \geq n .
$$

We also assume that $K \in \mathbb{R}^{d \times d}$, and that $L, \Lambda \in \mathbb{R}^{d \times d}$ satisfies

$$
L L^{\mathrm{T}} \geq(n+1) \Lambda \Lambda^{\mathrm{T}} .
$$

Condition (2.4) ensures $Y>0$ almost everywhere on $(t, \omega)$, which was established by Mayerhofer et al. (2011) using a generalized form of SDE, including a jump martingale part. The $\mathbb{S}_{++}^{d}$-valued process $Y$ is a stochastic factor process, which linearly depends on the covariance structure of $(S, Y)$ as

$$
\begin{aligned}
\frac{\mathrm{d}\left\langle S^{i}, S^{j}\right\rangle_{t}}{S_{t}^{i} S_{t}^{j}}= & \left(\Sigma Y_{t} \Sigma^{\mathrm{T}}\right)_{i j} \mathrm{~d} t \quad 1 \leq i, j \leq n, \\
\frac{\mathrm{d}\left\langle S^{i}, Y^{j k}\right\rangle_{t}}{S_{t}^{i}}= & \left\{\left(\Sigma Y_{t}\right)_{i j}(\Lambda \rho)_{k}+\left(\Sigma Y_{t}\right)_{i k}(\Lambda \rho)_{j}\right\} \mathrm{d} t \\
& 1 \leq i \leq n, 1 \leq j, k \leq d, \\
\mathrm{~d}\left\langle Y^{i j}, Y^{k l}\right\rangle_{t}= & \left\{Y_{t}^{i k}\left(\Lambda \Lambda^{\mathrm{T}}\right)_{j l}+Y_{t}^{i l}\left(\Lambda \Lambda^{\mathrm{T}}\right)_{j k}+Y_{t}^{j k}\left(\Lambda \Lambda^{\mathrm{T}}\right)_{i l}\right. \\
& \left.+Y_{t}^{j l}\left(\Lambda \Lambda^{\mathrm{T}}\right)_{i k}\right\} \mathrm{d} t 1 \leq i, j, k, l \leq d,
\end{aligned}
$$

as well as on the interest rate

$$
r\left(Y_{t}\right):=r_{0}+\operatorname{tr}\left(R_{1} Y_{t}\right),
$$

and on the so-called risk premium of $S$,

$$
\mu\left(Y_{t}\right)-r\left(Y_{t}\right) \mathbf{1}=\left(\Sigma Y_{t} \Sigma^{\mathrm{T}}\right) \lambda .
$$

Remark 2.1 From (2.6), we see that the interest rate process $\left(r\left(Y_{t}\right)\right)_{t>0}$ is included in the so-called affine class: $r\left(Y_{t}\right)$ is an affine function of $Y_{t}$ and the process $Y$, whose infinitesimal generator is given by

$$
\mathbb{L} f:=2 \operatorname{tr}\left(y D \Lambda \Lambda^{\mathrm{T}} D\right) f+\operatorname{tr}\left(\left(L L^{\mathrm{T}}+K y+y K^{\mathrm{T}}\right) D f\right),
$$

where $f \in C^{2}\left(\mathbb{S}^{d}\right)$ and

$$
D:=\left(\partial_{y_{i j}}\right)_{1 \leq i, j \leq d}
$$

is indeed an affine diffusion. To review affine processes and their financial applications, see, for example, [21], [22], and the references therein.

Remark 2.2 The condition (2.7) on the structure of the risk-premium vector is rewritten as

$$
\lambda=\left(\Sigma Y_{t} \Sigma^{\mathrm{T}}\right)^{-1}\left\{\mu\left(Y_{t}\right)-r\left(Y_{t}\right) \mathbf{1}\right\} .
$$

So we interpret that the so-called mean-variance term in portfolio optimization theory is assumed to be constant.

The following are concrete examples of setting up (2.1) and (2.2).

Example 2.1 (Stochastic Covariance) Let

$$
d=n, R_{1}=0 \text {, and } \Sigma=I_{n} .
$$

Concretely, we have

$$
\begin{aligned}
& \mathrm{d} S_{t}^{0}=r_{0} S_{t}^{0} \mathrm{~d} t, \quad S_{0}^{0}=1, \\
& \mathrm{~d} S_{t}=\operatorname{diag}\left(S_{t}\right)\left\{\left(r_{0} 1+Y_{t} \lambda\right) \mathrm{d} t+\sqrt{Y_{t}} \mathrm{~d} w_{t}\right\}, S_{0} \in \mathbb{R}_{++}^{n}
\end{aligned}
$$

with the third equation in (2.1). $Y$ describes the infinitesimal covariance and the risk premium of $S$ as

$$
Y_{t}^{i j} \mathrm{~d} t=\frac{\mathrm{d}\left\langle S^{i}, S^{j}\right\rangle_{t}}{S_{t}^{i} S_{t}^{j}} \quad \text { for } 1 \leq i, j \leq n
$$

and

$$
\mu\left(Y_{t}\right)-r_{0} \mathbf{1}=Y_{t} \lambda .
$$

This is exactly the model employed in Section 1 of [18] to study expected CRRA-utility maximization of terminal wealth.

Example 2.2 (Stochastic Covariance and Interest Rate) We present a slight generalization of Example 2.1 to include stochasticity of interest rates. Let

$$
d=n+1, R_{1}=\left(r_{i j}\right)_{1 \leq i, j \leq d} \text {, and } \Sigma=\left(I_{n}, 0\right),
$$

where we set $r_{i j}=0$ if $1 \leq i, j \leq n$. Then, letting

$$
Y_{1}(t):=\left(Y_{t}^{i j}\right)_{1 \leq i, j \leq n}, \quad Y_{2}(t):=\left(Y_{t}^{d 1}, \cdots, Y_{t}^{d d}\right)^{\mathrm{T}},
$$

and $\tilde{r}_{d}:=\left(r_{d 1}, 2 r_{d 2}, \cdots, 2 r_{d d}\right)^{\mathrm{T}} \in \mathbb{R}^{d}$, we see that

$$
r\left(Y_{2}(t)\right)=r_{0}+\tilde{r}_{d}^{\mathrm{T}} Y_{2}(t)
$$

is the risk-free interest rate with the latent factor $Y_{2}(t)$ and that

$$
\begin{aligned}
& \mathrm{d} S_{t}=\operatorname{diag}\left(S_{t}\right)\left[\left\{r\left(Y_{2}(t)\right) \mathbf{1}+Y_{1}(t) \lambda\right\} \mathrm{d} t+\sqrt{Y_{1}(t)} \mathrm{d} w_{t}\right], \\
& S_{0} \in \mathbb{R}_{++}^{n},
\end{aligned}
$$

where $Y_{1}(t)$ describes the infinitesimal covariance and 
the risk premium of $S$ as

and

$$
Y_{t}^{i j} \mathrm{~d} t=\frac{\mathrm{d}\left\langle S^{i}, S^{j}\right\rangle_{t}}{S_{t}^{i} S_{t}^{j}} \text { for } 1 \leq i, j \leq n
$$

$$
\mu\left(Y_{t}\right)-r\left(Y_{2}(t)\right) \mathbf{1}=Y_{1}(t) \lambda .
$$

\section{Example 2.3 (Cox-Ingersoll-Ross Interest Rate} Factor) Let

$$
d=n=1, r_{0}=0 \text { and } R_{1}=1 .
$$

Then, we see

$$
\begin{aligned}
& \mathrm{d} S_{t}^{0}=Y_{t} S_{t}^{0} \mathrm{~d} t, \quad S_{0}^{0}=1, \\
& \mathrm{~d} S_{t}=S_{t}\left\{\left(1+\Sigma^{2} \lambda\right) Y_{t} \mathrm{~d} t+\Sigma \sqrt{Y_{t}} \mathrm{~d} w_{t}\right\}, \quad S_{0}>0, \\
& \mathrm{~d} Y_{t}=\left(L^{2}+2 K Y_{t}\right) \mathrm{d} t+2 \Lambda \sqrt{Y_{t}} \mathrm{~d} B_{t}, \quad Y_{0}>0 .
\end{aligned}
$$

This financial market model with Cox-Ingersoll-Ross's interest rate $Y$ is treated in [23] to study (1.3).

Under the financial market model comprising (2.1) and (2.2) with the assumptions (2.3) and (2.4), we are interested in treating the risk-sensitive asset management problem (1.3).

\subsection{Deriving the HJB Equation}

To tackle (1.3), we employ a dynamic programming approach: Recall that wealth process (1.1) of a selffinancing investor, combined with (2.1), is rewritten as $\mathrm{d} X_{t}^{\pi}=X_{t}^{\pi} \pi_{t}^{\mathrm{T}}\left[\left\{\mu\left(Y_{t}\right)-r\left(Y_{t}\right) \mathbf{1}\right\} \mathrm{d} t+\Sigma \sqrt{Y_{t}} \mathrm{~d} w_{t}+r\left(Y_{t}\right) \mathrm{d} t\right]$, $X_{0}^{\pi}=x$.

So, we see

$$
\begin{aligned}
& \mathrm{d}\left(X_{t}^{\pi}\right)^{-\gamma}=\left(X_{t}^{\pi}\right)^{-\gamma}\left[-\gamma \ell\left(Y_{t}, \pi_{t}\right) \mathrm{d} t-\gamma \pi_{t}^{\mathrm{T}} \Sigma \sqrt{Y_{t}} \mathrm{~d} w_{t}\right], \\
& \left(X_{0}^{\pi}\right)^{-\gamma}=x^{-\gamma},
\end{aligned}
$$

where we set

$$
\ell(y, \pi):=r(y)+\pi^{\mathrm{T}} \Sigma y \Sigma^{\mathrm{T}} \lambda-\frac{1+\gamma}{2} \pi^{\mathrm{T}} \Sigma y \Sigma^{\mathrm{T}} \pi .
$$

Hence, we have

$$
\left(X_{T}^{\pi}\right)^{-\gamma}=x^{-\gamma} M_{T}^{(\gamma \pi)} \exp \left\{-\gamma \int_{0}^{T} \ell\left(Y_{t}, \pi_{t}\right) \mathrm{d} t\right\},
$$

where we define

$$
M_{t}^{(\gamma \pi)}:=\exp \left(-\gamma \int_{0}^{t} \pi_{u}^{\mathrm{T}} \Sigma \sqrt{Y_{u}} \mathrm{~d} w_{u}-\frac{\gamma^{2}}{2} \int_{0}^{t} \pi_{u}^{\mathrm{T}} \Sigma Y_{u} \Sigma^{\mathrm{T}} \pi_{u} \mathrm{~d} u\right) .
$$

Let

$$
\mathscr{L}_{T}^{1}:=\left\{\pi \in \mathscr{L}_{2, T}^{n} ;\left(M_{t}^{(\gamma \pi)}\right)_{t \in[0, T]} \text { is a martingale }\right\} .
$$

For $\pi \in \mathscr{A}_{T}^{1}$, we define the probability measure $\tilde{\mathbb{P}}_{T}^{(\gamma \pi)}$ on $\left(\Omega, \mathcal{F}_{T}\right)$ by the formula

$$
\left.\frac{\mathrm{d} \tilde{\mathbb{P}}_{T}^{(\gamma \pi)}}{\mathrm{d} \mathbb{P}}\right|_{\mathcal{F}_{t}}:=M_{t}^{(\gamma \pi)}, \quad t \in[0, T] .
$$

By Cameron-Martin-Maruyama-Girsanov's theorem, we see that the $\mathbb{R}^{n \times n}$-valued process $\tilde{B}:=\left(\tilde{B}_{t}\right)_{t \in[0, T]}$, defined by

$$
\tilde{B}_{t}:=B_{t}+\gamma\left(\int_{0}^{t} \sqrt{Y_{u}} \Sigma^{\mathrm{T}} \pi_{u} \mathrm{~d} u\right) \rho^{\mathrm{T}},
$$

is a $\left(\tilde{\mathbb{P}}_{T}^{(\gamma \pi)}, \mathcal{F}_{t}\right)$-Brownian motion. Moreover, we see that $Y$ has the $\mathbb{\mathbb { P }}_{T}^{(\gamma \pi)}$-dynamics

$$
\begin{aligned}
\mathrm{d} Y_{t}= & \left(L L^{\mathrm{T}}+K Y_{t}+Y_{t} K^{\mathrm{T}}\right) \mathrm{d} t \\
& +\sqrt{Y_{t}}\left(\mathrm{~d} \tilde{B}_{t}-\gamma \sqrt{Y_{t}} \Sigma^{\mathrm{T}} \pi_{t} \rho^{\mathrm{T}} \mathrm{d} t\right) \Lambda^{\mathrm{T}} \\
& +\Lambda\left(\mathrm{d} \tilde{B}_{t}-\gamma \sqrt{Y_{t}} \Sigma^{\mathrm{T}} \pi_{t} \rho^{\mathrm{T}} \mathrm{d} t\right)^{\mathrm{T}} \sqrt{Y_{t}} \\
= & \left\{L L^{\mathrm{T}}+\left(K-\gamma \Lambda \rho \pi_{t}^{\mathrm{T}} \Sigma\right) Y_{t}+Y_{t}\left(K-\gamma \Lambda \rho \pi_{t}^{\mathrm{T}} \Sigma\right)^{\mathrm{T}}\right\} \mathrm{d} t \\
& +\sqrt{Y_{t}} \mathrm{~d} \tilde{B}_{t} \Lambda^{\mathrm{T}}+\Lambda \mathrm{d} \tilde{B}_{t} \sqrt{Y_{t}} .
\end{aligned}
$$

Recall that, for $\pi \in \mathscr{A}_{T}^{1}$, we have

$$
\begin{aligned}
& \frac{1}{(-\gamma)} \log \mathbb{E}\left(X_{T}^{\pi}\right)^{-\gamma} \\
& =\log x+\frac{1}{(-\gamma)} \log \tilde{\mathbb{E}}_{T}^{(\gamma \pi)} \exp \left\{-\gamma \int_{0}^{T} \ell\left(Y_{t}, \pi_{t}\right) \mathrm{d} t\right\},
\end{aligned}
$$

where $\tilde{\mathbb{P}}_{T}^{(\gamma \pi)}(\cdot)$ denotes the expectation with respect to $\tilde{\mathbb{P}}_{T}^{(\gamma \pi)}$. We now consider, for $0 \leq t \leq T<\infty$,

$$
\bar{V}_{t}^{(T)}:=\underset{\pi \in \lambda_{t, T}}{\operatorname{ess} \sup _{T}} \frac{1}{(-\gamma)} \log \tilde{\mathbb{E}}_{T}^{(\gamma \pi)}\left[\mathrm{e}^{-\gamma \int_{t}^{T}\left(Y_{u}, \pi_{u}\right) \mathrm{d} u} \mid \mathcal{F}_{t}\right]
$$

where

$$
\mathscr{A}_{t, T}^{1}:=\left\{\pi 1_{[t, T]} ; \pi \in \mathscr{A}_{T}^{1}\right\} .
$$

The associated HJB equation is written as

$$
\begin{aligned}
-\partial_{t} V= & 2 \operatorname{tr}\left(y D\left(\Lambda \Lambda^{\mathrm{T}} D\right)\right) V-2 \gamma \operatorname{tr}\left(y D V \Lambda \Lambda^{\mathrm{T}} D V\right) \\
& +\sup _{\pi \in \mathbb{R}^{n}}\left[\operatorname { t r } \left(\left\{L L^{\mathrm{T}}+\left(K-\gamma \Lambda \rho \pi^{\mathrm{T}} \Sigma\right) y\right.\right.\right. \\
& \left.\left.\left.+y\left(K-\gamma \Lambda \rho \pi^{\mathrm{T}} \Sigma\right)^{\mathrm{T}}\right\} D V\right)+\ell(y, \pi)\right] \\
V(T, y) & =0 .
\end{aligned}
$$

By direct calculation, we can see the following.

Lemma 2.1 1) If $d \leq n$ and $\Sigma^{\mathrm{T}} \Sigma>0$, then $H J B$ Equation (2.13) is rewritten as

$$
\begin{aligned}
-\partial_{t} V= & 2 \operatorname{tr}\left(y D\left(\Lambda \Lambda^{\mathrm{T}} D\right)\right) V-\operatorname{tr}\left(y D V \Lambda N^{-1} \Lambda^{\mathrm{T}} D V\right) \\
& +\operatorname{tr}\left(\left(K_{1} y+y K_{1}^{\mathrm{T}}+L L^{\mathrm{T}}\right) D V\right)+\operatorname{tr}(y A)+r_{0}, \\
V(T, y) & =0,
\end{aligned}
$$


where we define

$$
\begin{aligned}
& N^{-1}:=2 \gamma\left(I-\frac{\gamma}{1+\gamma} \rho \rho^{\mathrm{T}}\right), \\
& K_{1}:=K-\frac{\gamma}{1+\gamma} \Lambda \rho \lambda^{\mathrm{T}} \Sigma, \\
& A:=\frac{1}{2(1+\gamma)} \Sigma^{\mathrm{T}} \lambda \lambda^{\mathrm{T}} \Sigma+R_{1} .
\end{aligned}
$$

The maximizer for (2.13) is given by

$$
\bar{\pi}^{(T)}(t, y):=\frac{1}{1+\gamma}\left\{\lambda-2 \gamma \Sigma\left(\Sigma^{\mathrm{T}} \Sigma\right)^{-1} D V(t, y) \Lambda \rho\right\} .
$$

2) If $d \geq n$ and $\Sigma \Sigma^{\mathrm{T}}>0$, then HJB equation (2.13) is rewritten as

$$
\begin{aligned}
-\partial_{t} V= & 2 \operatorname{tr}\left(y D\left(\Lambda \Lambda^{\mathrm{T}} D\right)\right) V-2 \gamma \operatorname{tr}\left(y D V \Lambda \Lambda^{\mathrm{T}} D V\right) \\
& +\frac{2 \gamma^{2}}{1+\gamma} \operatorname{tr}\left(y \Sigma^{\mathrm{T}}\left(\Sigma y \Sigma^{\mathrm{T}}\right)^{-1} \Sigma y D V \Lambda \rho \rho^{\mathrm{T}} \Lambda^{\mathrm{T}} D V\right) \\
& +\operatorname{tr}\left(\left(K_{1} y+y K_{1}^{\mathrm{T}}+L L^{\mathrm{T}}\right) D V\right)+\operatorname{tr}(y A)+r_{0}, \\
V(T, y)= & 0,
\end{aligned}
$$

where $K_{1} \in \mathbb{R}^{n \times d}$ and $A \in \mathbb{R}^{d \times d}$ are given by (2.15). The maximizer for (2.13) is given by

$$
\bar{\pi}^{(T)}(t, y):=\frac{1}{1+\gamma}\left\{\lambda-2 \gamma\left(\Sigma y \Sigma^{\mathrm{T}}\right)^{-1} \Sigma y D V(t, y) \Lambda \rho\right\} .
$$

\section{Results}

With the help of Lemma 2.1, it is straightforward to see the following.

Proposition 3.1 (Solution to the HJB equation) 1) If $d \leq n$, then

$$
\hat{V}^{(T)}(t, y):=\operatorname{tr}\left(P^{(T)}(t) y\right)+p^{(T)}(t)
$$

solves (2.13), or equivalently (2.14). Here, $P:=P^{(T)}:[0, T] \rightarrow \mathbb{S}^{d}$ and $p:=p^{(T)}:[0, T] \rightarrow \mathbb{R}$ solve the following system of ordinary differential equations:

$$
\begin{aligned}
& \frac{\mathrm{d}}{\mathrm{d} t} P-P \Lambda N^{-1} \Lambda^{\mathrm{T}} P+P K_{1}+K_{1}^{\mathrm{T}} P+A=0, P(T)=0, \\
& \frac{\mathrm{d}}{\mathrm{d} t} p+\operatorname{tr}\left(P L L^{\mathrm{T}}\right)+r_{0}=0, p(T)=0 .
\end{aligned}
$$

2) If $d \geq n$, then

$$
\hat{V}^{(T)}(t, y):=\operatorname{tr}\left(\Sigma^{\mathrm{T}} P^{(T)}(t) \Sigma y\right)+p^{(T)}(t)
$$

solves (2.13), or equivalently (2.16). Here, $P:=P^{(T)}:[0, T] \rightarrow \mathbb{S}^{n}$ and $p:=p^{(T)}:[0, T] \rightarrow \mathbb{R}$ solve the following system of ordinary differential equations:

$$
\begin{aligned}
& \frac{\mathrm{d}}{\mathrm{d} t} P-P \Sigma \Lambda N^{-1} \Lambda^{\mathrm{T}} \Sigma^{\mathrm{T}} P+P \Sigma K_{1} \Sigma^{\mathrm{T}}\left(\Sigma \Sigma^{\mathrm{T}}\right)^{-1} \\
& +\left(\Sigma \Sigma^{\mathrm{T}}\right)^{-1} \Sigma K_{1}^{\mathrm{T}} \Sigma^{\mathrm{T}} P+\left(\Sigma \Sigma^{\mathrm{T}}\right)^{-1} \Sigma A \Sigma^{\mathrm{T}}\left(\Sigma \Sigma^{\mathrm{T}}\right)^{-1}=0, P(T)=0, \\
& \frac{\mathrm{d}}{\mathrm{d} t} p+\operatorname{tr}\left(P \Sigma L L^{\mathrm{T}} \Sigma^{\mathrm{T}}\right)+r_{0}=0, p(T)=0 .
\end{aligned}
$$

Using this proposition, we obtain the following.

\section{Theorem 3.1 (Verification and optimal strategy)} Define the filtration $\left(\mathcal{F}_{t}^{B}\right)_{t \in[0, T]}$ by $\mathcal{F}_{t}^{B}:=\sigma\left(B_{u} ; u \in[0, t]\right)$. Let

$$
\mathscr{A}_{T}^{B b}:=\left\{\begin{array}{l|l}
\left(\pi_{t}\right)_{t \in[0, T]} & \begin{array}{l}
\text { bounded, } \\
\mathcal{F}_{t}^{B} \text {-progressively measurable }
\end{array}
\end{array}\right\}
$$

and consider (1.3) with $\mathscr{A}_{T}:=\mathscr{A}_{T}^{B b}$. Then, the following assertions hold.

1) If $d \leq n$, then $\hat{\pi}^{(T)}:=\left(\hat{\pi}_{t}^{(T)}\right)_{t \in[0, T]}$, defined by

$$
\hat{\pi}_{t}^{(T)}:=\frac{1}{1+\gamma} \lambda-\frac{2 \gamma}{1+\gamma} \Sigma\left(\Sigma^{\mathrm{T}} \Sigma\right)^{-1} P^{(T)}(t) \Lambda \rho,
$$

is optimal for (1.3). It holds that

$$
\Gamma_{T}(\gamma)=\frac{1}{T}\left\{\log x+\hat{V}^{(T)}\left(0, Y_{0}\right)\right\} .
$$

2) If $d \geq n$, then $\hat{\pi}^{(T)}:=\left(\hat{\pi}_{t}^{(T)}\right)_{t \in[0, T]}$, defined by

$$
\hat{\pi}_{t}^{(T)}:=\frac{1}{1+\gamma} \lambda-\frac{2 \gamma}{1+\gamma} P^{(T)}(t) \Sigma \Lambda \rho,
$$

is optimal for (1.3). The relation (3.7) holds.

The proof of the above theorem is given in Subsection 4.2 after preparing lemmas in Subsection 4.1.

\section{Proofs}

\subsection{Lemmas for Exponential Martingale}

We prepare the following two lemmas.

Lemma 4.1 Let $(F, f): \mathbb{R}_{+} \times \Omega \rightarrow \mathbb{R}^{d \times d} \times \mathbb{R}^{d}$ be $\mathcal{F}_{t}^{B}$-progressively measurable so that $\int_{0}^{T}\left(\left|F_{t}\right|^{2}+|f|_{t}^{2}\right) \mathrm{d} t<\infty$ almost surely for all $T>0$.

Define

$$
\begin{gathered}
\mathcal{E}_{1}(t)=\mathcal{E}_{1}(t ; B):=\exp \left\{\int_{0}^{t} \operatorname{tr}\left(F_{u} \mathrm{~d} B_{u}\right)-\frac{1}{2} \int_{0}^{t} \operatorname{tr}\left(F_{u} F_{u}^{\mathrm{T}}\right) \mathrm{d} u\right\}, \\
\mathcal{E}_{2}(t)=\mathcal{E}_{2}(t ; B, z):=\exp \left\{\int_{0}^{t} f_{u}^{\mathrm{T}} \mathrm{d} z_{u}-\frac{1}{2} \int_{0}^{t}\left|f_{u}\right|^{2} \mathrm{~d} u\right\} .
\end{gathered}
$$

Then, $\mathcal{E}_{1} \mathcal{E}_{2}$ is an $\mathcal{F}_{t}$-martingale if and only if $\mathcal{E}_{1}$ is an $\mathcal{F}_{t}$-martingale.

Proof. Denote $\mathcal{F}_{t}^{z}:=\sigma\left(z_{u} ; u \in[0, t]\right)$. For 
$0 \leq s \leq t \leq T$, we have

$$
\begin{aligned}
& \mathbb{E}\left[\mathcal{E}_{1}(t) \mathcal{E}_{2}(t) \mid \mathcal{F}_{s}\right] \\
& =\mathbb{E}\left[\mathbb{E}\left[\mathcal{E}_{1}(t) \mathcal{E}_{2}(t) \mid \mathcal{F}_{t}^{B} \vee \mathcal{F}_{s}^{z}\right] \mid \mathcal{F}_{s}\right] \\
& =\mathcal{E}_{2}(s) \mathbb{E}\left[\mathcal{E}_{1}(t) \mathbb{E}\left[\mathcal{E}_{2}(t) \mathcal{E}_{2}^{-1}(s) \mid \mathcal{F}_{t}^{B} \vee \mathcal{F}_{s}^{z}\right] \mid \mathcal{F}_{s}\right] \\
& =\mathcal{E}_{2}(s) \mathbb{E}\left[\mathcal{E}_{1}(t) \mathbb{E}\left[\mathcal{E}_{2}(t) \mathcal{E}_{2}^{-1}(s) \mid \mathcal{F}_{t}^{B}\right] \mid \mathcal{F}_{s}\right] \\
& =\mathcal{E}_{2}(s) \mathbb{E}\left[\left.\mathcal{E}_{1}(t) \mathbb{E}\left[\mathcal{E}_{2}(t, l) \mathcal{E}_{2}^{-1}(s, l)\right]\right|_{l=B} \mid \mathcal{F}_{s}\right] \\
& =\mathcal{E}_{2}(s) \mathbb{E}\left[\mathcal{E}_{1}(t) \mid \mathcal{F}_{s}\right] .
\end{aligned}
$$

Lemma 4.2 Let $F: \mathbb{R}_{+} \times \mathbb{S}^{d} \times \Omega \rightarrow \mathbb{R}^{d \times d}$ satisfy the following: for each $t \geq 0, \quad(s, y, \omega) \mapsto F(s, y, \omega)$ is $\mathcal{B}([0, t]) \otimes \mathcal{B}\left(\mathcal{B}^{d}\right) \otimes \mathcal{F}_{t} / \mathcal{B}\left(\mathbb{R}^{d \times d}\right)$-measurable, and

$\left|F F^{\mathrm{T}}(t, y, \omega)\right| \leq C_{0}(t, \omega)|y|$ for all $(t, y, \omega) \in \mathbb{R}_{+} \times \mathbb{S}^{d} \times \Omega$

with some bounded $C_{0}$, where we write $|y|:=\sqrt{\operatorname{tr}\left(y^{2}\right)}$ for $y \in \mathbb{S}^{d}$. Then, the process $\mathcal{E}:=\left(\mathcal{E}_{t}\right)_{t>0}$, defined by

$$
\mathcal{E}_{t}:=\exp \left\{\int_{0}^{t} \operatorname{tr}\left(F\left(u, Y_{u}\right) \mathrm{d} B_{u}\right)-\frac{1}{2} \int_{0}^{t} \operatorname{tr}\left(F F^{\mathrm{T}}\right)\left(u, Y_{u}\right) \mathrm{d} u\right\},
$$

is a martingale.

Proof. The lemma follows from Lemma 4.1.5 of [24], an extension of Lemma 4.1.1 of [25]. Below, we reproduce the proof for self-containedness. Note that it suffices to show

$$
\begin{aligned}
& \mathbb{E} \mathcal{E}_{t}=1 \text { for each } t>0 \text {. } \\
& \mathrm{d}\left(\frac{\mathcal{E}_{t} \phi\left(Y_{t}\right)}{1+\epsilon \mathcal{E}_{t} \phi\left(Y_{t}\right)}\right)=\frac{\phi\left(Y_{t}\right) \mathrm{d} \mathcal{E}_{t}+\mathcal{E}_{t} \mathrm{~d} \phi\left(Y_{t}\right)}{\left(1+\epsilon \mathcal{E}_{t} \phi\left(Y_{t}\right)\right)^{2}}-\epsilon \frac{\phi\left(Y_{t}\right)^{2} \mathrm{~d}\langle\mathcal{E}\rangle_{t}+2 \phi\left(Y_{t}\right) \mathcal{E}_{t} \mathrm{~d}\langle\mathcal{E}, \phi(Y)\rangle_{t}+\mathcal{E}_{t}^{2} \mathrm{~d}\langle\phi(Y)\rangle_{t}}{\left(1+\epsilon \mathcal{E}_{t} \phi\left(Y_{t}\right)\right)^{3}}=: \mathrm{d} M_{t}+\mathrm{d} A_{t},
\end{aligned}
$$

Recall that $(t, \omega) \mapsto F\left(t, Y_{t}(\omega), \omega\right)$ is progressively measurable. The proof of (4.1) consists of several steps.

First, writing $\phi(y):=|y|$, we recall that

$$
\mathbb{L} \phi \leq C_{1} \phi \text { with some } C_{1} \in \mathbb{R}_{++},
$$

where $\mathbb{L}$ is defined by (2.8). From this, we can check that

$$
\mathbb{E} \phi\left(Y_{t}\right) \leq \phi\left(Y_{0}\right) \mathrm{e}^{C_{2} t}
$$

for each $t \geq 0$ with some constant $C_{2}$. Also, we can check that

$$
\mathcal{E}_{t} \phi\left(Y_{t}\right) \in L^{1}(\mathbb{P}) \text { for each } t \geq 0 .
$$

This follows from the relation

$$
\mathbb{E}\left[\frac{\mathcal{E}_{t} \phi\left(Y_{t}\right)}{1+\epsilon \mathcal{E}_{t} \phi\left(Y_{t}\right)}\right] \leq\left|Y_{0}\right| \mathrm{e}^{C_{3} t},
$$

where $\epsilon>0$ is arbitrary and the constant $C_{3}$ is independent of $\epsilon$. Indeed, in (4.4), letting $\epsilon \downarrow 0$ and using Fatou's lemma, (4.3) is deduced. To see (4.4), use (2.1) and Itô's formula to deduce

$$
\begin{gathered}
\mathrm{d} \mathcal{E}_{t}=\mathcal{E}_{t} \operatorname{tr}\left(F\left(t, Y_{t}\right) \mathrm{d} B_{t}\right), \\
\mathrm{d} \phi\left(Y_{t}\right)=\operatorname{tr}\left(D \phi\left(Y_{t}\right)\left(\sqrt{Y_{t}} \mathrm{~d} B_{t} \Lambda^{\mathrm{T}}+\Lambda \mathrm{d} B_{t}^{\mathrm{T}} \sqrt{Y_{t}}\right)\right)+\mathbb{L} \phi\left(Y_{t}\right) \mathrm{d} t,
\end{gathered}
$$

where we use notation (2.8) and (2.9). From these, we

where

$$
M_{t}:=\int_{0}^{t} \frac{\mathcal{E}_{u}}{\left(1+\epsilon \mathcal{E}_{u} \phi\left(Y_{u}\right)\right)^{2}} \times\left\{\phi\left(Y_{u}\right) \operatorname{tr}\left(F\left(u, Y_{u}\right) \mathrm{d} B_{u}\right)+\frac{1}{\phi\left(Y_{u}\right)} \operatorname{tr}\left(Y_{u}^{3 / 2} \mathrm{~d} B_{u} \Lambda^{\mathrm{T}}+\Lambda \mathrm{d} B_{u}^{\mathrm{T}} Y_{u}^{3 / 2}\right)\right\}
$$

is the local-martingale part and $\left(A_{t}\right)_{t \geq 0}$ is the bounded-variation part, which satisfies

$$
\begin{aligned}
& A_{t} \leq \frac{\phi\left(Y_{0}\right)}{1+\epsilon \phi\left(Y_{0}\right)}+\int_{0}^{t} \frac{\mathcal{E}_{u} \mathbb{L} \phi\left(Y_{u}\right)}{\left(1+\epsilon \mathcal{E}_{u} \phi\left(Y_{u}\right)\right)^{2}} \mathrm{~d} u \\
& +C_{4} \int_{0}^{t} \frac{\epsilon \mathcal{E}_{u}^{2}}{\left(1+\epsilon \mathcal{E}_{u} \phi\left(Y_{u}\right)\right)^{3}}\left\{\phi\left(Y_{u}\right)^{2} \operatorname{tr}\left(F F^{\mathrm{T}}\left(u, Y_{u}\right)\right)+2 \phi\left(Y_{u}\right)\left|\operatorname{tr}\left(F\left(u, Y_{u}\right) Y_{u}^{1 / 2}\right)\right|+\frac{\operatorname{tr}\left(Y_{u}^{3}\right)}{\phi\left(Y_{u}\right)^{2}}\right\} \mathrm{d} u
\end{aligned}
$$

with some constant $C_{4}$, independent of $\epsilon$. We can check that $\mathbb{E}\langle M\rangle_{t}<\infty$; hence, $M$ is a square-integrable martingale. Further, using (4.2) and recalling that $|A B| \leq|A||B|$ for conformable matrices $A$ and $B$, we can check that

$$
A_{t} \leq A_{0}+C_{5} \int_{0}^{t} \frac{\mathcal{E}_{s} \phi\left(Y_{s}\right)}{1+\epsilon \mathcal{E}_{s} \phi\left(Y_{s}\right)} \mathrm{d} s
$$

with some positive constant $C_{5}$, independent of $\epsilon>0$. So, taking the expectation, we deduce that

$$
\mathbb{E}\left[\frac{\mathcal{E}_{t} \phi\left(Y_{t}\right)}{1+\epsilon \mathcal{E}_{t} \phi\left(Y_{t}\right)}\right] \leq \frac{\phi\left(Y_{0}\right)}{1+\epsilon \phi\left(Y_{0}\right)}+C_{5} \int_{0}^{t} \mathbb{E}\left[\frac{\mathcal{E}_{s} \phi\left(Y_{s}\right)}{1+\epsilon \mathcal{E}_{s} \phi\left(Y_{s}\right)}\right] \mathrm{d} s
$$

and that (4.4) follows from Gronwall's inequality.

Next, use Itô's formula for the following computation: 


$$
\mathrm{d}\left(\frac{\mathcal{E}_{t}}{1+\epsilon \mathcal{E}_{t}}\right)=\frac{\mathrm{d} \mathcal{E}_{t}}{\left(1+\epsilon \mathcal{E}_{t}\right)^{2}}-\epsilon \frac{\mathrm{d}\langle\mathcal{E}\rangle_{t}}{\left(1+\epsilon \mathcal{E}_{t}\right)^{3}} .
$$

Here, we see that

$$
\mathbb{E} \int_{0}^{t} \frac{\mathrm{d}\langle\mathcal{E}\rangle_{s}}{\left(1+\epsilon \mathcal{E}_{s}\right)^{4}} \leq C_{6} \mathbb{E} \int_{0}^{t} \operatorname{tr}\left(F F^{\mathrm{T}}\left(s, Y_{s}\right)\right) \mathrm{d} s<\infty
$$

with some constant $C_{6}$; hence, the first term of the righthand side of (4.5) is a square-intergrable martingale. Also, we can deduce that

$$
\epsilon \int_{0}^{t} \frac{\mathrm{d}\langle\mathcal{E}\rangle_{s}}{\left(1+\epsilon \mathcal{E}_{s}\right)^{3}} \leq C_{7} \int_{0}^{t} \mathcal{E}_{s}\left|Y_{s}\right| \mathrm{d} s
$$

where $C_{7}$ is a positive constant, independent of $\epsilon$. Taking the expectation, we see

$$
\mathbb{E} \frac{\mathcal{E}_{t}}{1+\epsilon \mathcal{E}_{t}}=\frac{1}{1+\epsilon}-\epsilon \mathbb{E} \int_{0}^{t} \frac{\mathrm{d}\langle\mathcal{E}\rangle_{s}}{\left(1+\epsilon \mathcal{E}_{s}\right)^{3}} .
$$

Letting $\epsilon \downarrow 0$ and using the dominated convergence theorem, we obtain (4.1).

\subsection{Proof of Theorem 3.1}

Let $\hat{V}:=\hat{V}^{(T)}:[0, T] \times \mathbb{S}_{++}^{d} \rightarrow \mathbb{R}$ be given by (3.1). Fix $(t, y) \in[0, T] \times \mathbb{S}_{++}^{d}$ and take $\pi \in \mathscr{A}_{T-t}^{B b}$. Using these, define

$$
\Phi_{s}(\pi):=M_{s}^{(\gamma \pi)} \exp \left[-\gamma\left\{\hat{V}\left(t+s, Y_{s}\right)+\int_{0}^{s} \ell\left(Y_{u}, \pi_{u}\right) \mathrm{d} u\right\}\right],
$$

where we use (2.10), (2.11), and the process $Y$ given by (2.1), and we set $Y_{0}=y \in \mathbb{S}_{++}^{d}$. Using Itô's formula, we see that

$$
\frac{\mathrm{d} \Phi_{s}(\pi)}{\Phi_{s}(\pi)}=\frac{\mathrm{d} \mathcal{E}_{s}(\pi)}{\mathcal{E}_{s}(\pi)}-\gamma\left(\mathcal{L}_{s}^{(\pi)} \hat{V}\right)\left(t+s, Y_{s}\right) \mathrm{d} s,
$$

where we define the process $\mathcal{E}(\pi):=\left(\mathcal{E}_{s}(\pi)\right)_{s \in[0, T-t]}$ by (see below)

Combining (4.6)-(4.8), we have, for $s \in[0, T-t]$,

$$
\begin{aligned}
& \Phi_{s}(\pi) \\
& =\mathcal{E}_{s}(\pi) \times \exp \left[-\gamma\left\{\hat{V}(t, y)+\int_{0}^{s}\left(\mathcal{L}_{s}^{(\pi)} \hat{V}\right)\left(t+u, Y_{u}\right) \mathrm{d} u\right\}\right] .
\end{aligned}
$$

Here, note that $\left(\mathcal{E}_{s}(\pi)\right)_{s \in[0, T-t]}$ is a martingale for any $\pi \in \mathscr{\mathscr { N }}_{T-t}^{B b}$ by using Lemma 4.1 and 4.2 and that $\left(\mathcal{L}_{s}^{(\pi)} \hat{V}\right)\left(t+s, Y_{s}\right) \leq 0 \quad$ almost everywhere on $(s, \omega) \in[0, T-t] \times \Omega$ since $\hat{V}$ solves HJB-equation (2.13). So we deduce that $\left(\Phi_{s}(\pi)\right)_{s \in[0, T-t]}$ is a submartingale for each $\pi \in \mathscr{A}_{T-t}^{B b}$. Taking the expectation, we see that

$$
\begin{aligned}
& \exp \{-\gamma \hat{V}(t, y)\} \leq \mathbb{E} \Phi_{T-t}(\pi) \\
& =\mathbb{E}_{T}^{(\gamma \pi)} \exp \left\{-\gamma \int_{0}^{T-t} \ell\left(Y_{u}, \pi_{u}\right) \mathrm{d} u\right\} .
\end{aligned}
$$

Thus, we see that

$$
\frac{1}{(-\gamma)} \log \mathbb{E}_{T}^{(\gamma \pi)} \exp \left\{-\gamma \int_{0}^{T-t} \ell\left(Y_{u}, \pi_{u}\right) \mathrm{d} u\right\} \leq \hat{V}(t, y)
$$

for any $\pi \in \mathscr{A}_{T-t}^{B b}$. Furthermore, if we define

$$
\tilde{\pi}:=\left(\tilde{\pi}_{s}\right)_{s \in[0, T-t]} \in \mathscr{A}_{T-t}^{B b} \text { by }
$$

$$
\tilde{\pi}_{s}:=\hat{\pi}_{t+s}^{(T)},
$$

then, we deduce that $\left(\mathcal{L}_{s}^{(\tilde{\pi})} \hat{V}\right)\left(t+s, Y_{s}\right)=0$ almost everywhere on $(s, \omega) \in[0, T-t] \times \Omega$, from which we see that $\left(\Phi_{s}(\tilde{\pi})\right)_{s \in[0, T-t]}$ is a martingale. Therefore, taking the expectation, we see that

$$
\begin{aligned}
& \exp \{-\gamma \hat{V}(t, y)\}=\mathbb{E} \Phi_{T-t}(\tilde{\pi}) \\
& =\mathbb{E}_{T}^{(\gamma \tilde{\pi})} \exp \left\{-\gamma \int_{0}^{T-t} \ell\left(Y_{u}, \tilde{\pi}_{u}\right) \mathrm{d} u\right\},
\end{aligned}
$$

that is,

$$
\frac{1}{(-\gamma)} \log \mathbb{E}_{T}^{(\gamma \tilde{\pi})} \exp \left\{-\gamma \int_{0}^{T-t} \ell\left(Y_{u}, \tilde{\pi}_{u}\right) \mathrm{d} u\right\}=\hat{V}(t, y) .
$$

Combining (4.9) and (4.10), we deduce that

$$
\hat{V}(t, y)=\sup _{\substack{\pi \in \cup_{T-t}^{B b} \\(-\gamma)}} \frac{1}{\log } \log \mathbb{E}_{T}^{(\gamma \pi)} \exp \left\{-\gamma \int_{0}^{T-t} \ell\left(Y_{u}, \pi_{u}\right) \mathrm{d} u\right\} .
$$

Thus, letting $t=0$ in (4.11), we have that

$$
\frac{\mathrm{d} \mathcal{E}_{s}(\pi)}{\mathcal{E}_{s}(\pi)}=-\gamma \operatorname{tr}\left(\sqrt{Y_{s}} \mathrm{~d} B_{s}\left\{2 \Lambda^{\mathrm{T}} D \hat{V}\left(t+s, Y_{s}\right)+\rho \pi_{s}^{\mathrm{T}} \Sigma\right\}\right)-\gamma \sqrt{1-|\rho|^{2}} \pi_{s}^{\mathrm{T}} \Sigma \sqrt{Y_{s}} \mathrm{~d} z_{s}, \quad \mathcal{E}_{0}(\pi)=1
$$

and write

$$
\begin{aligned}
\left(\mathcal{L}_{s}^{(\pi)} \hat{V}\right)(t+s, y):= & \partial_{s} \hat{V}(t+s, y)+2 \operatorname{tr}\left(y D \Lambda \Lambda^{\mathrm{T}} D\right) \hat{V}(t+s, y)-2 \gamma \operatorname{tr}\left(y D \hat{V} \Lambda \Lambda^{\mathrm{T}} D \hat{V}\right)(t+s, y) \\
& +\operatorname{tr}\left(\left\{L L^{\mathrm{T}}+\left(K-\gamma \Lambda \rho \pi_{s}^{\mathrm{T}} \Sigma\right) y+y\left(K-\gamma \Lambda \rho \pi_{s}^{\mathrm{T}} \Sigma\right)^{\mathrm{T}}\right\} D \hat{V}\right)(t+s, y)+\ell\left(y, \pi_{s}\right) .
\end{aligned}
$$




$$
\begin{aligned}
\hat{V}^{(T)}\left(0, Y_{0}\right) & =\sup _{\substack{\varkappa_{T} \\
T}} \frac{1}{(-\gamma)} \log \mathbb{E}_{T}^{(\gamma \pi)} \exp \left\{-\gamma \int_{0}^{T} \ell\left(Y_{u}, \pi_{u}\right) \mathrm{d} u\right\} \\
& =\frac{1}{(-\gamma)} \log \mathbb{E}_{T}^{\left(\gamma \hat{\pi}^{(T)}\right)} \exp \left\{-\gamma \int_{0}^{T} \ell\left(Y_{u}, \hat{\pi}_{u}^{(T)}\right) \mathrm{d} u\right\} .
\end{aligned}
$$

(3.7) follows from relation (2.12).

\section{REFERENCES}

[1] T. R. Bielecki and S. R. Pliska, "Risk Sensitive Dynamic Asset Management," Applied Mathematics and Optimization, Vol. 39, No. 3, 1999, pp. 337-360. doi:10.1007/s002459900110

[2] T. R. Bielecki and S. R. Pliska, "Risk Sensitive Intertemporal CAPM, with Application to Fixed-Income Management," IEEE Transactions on Automat. Control, Vol. 49, No. 3, 2004, pp. 420-432. doi:10.1109/TAC.2004.824470

[3] M. H. A. Davis and S. Lleo, "Risk-Sensitive Benchmarked Asset Management," Quantitative Finance, Vol. 8, No. 4, 2008, pp. 415-426.

[4] W. H. Fleming and S. J. Sheu, "Risk-Sensitive Control and an Optimal Investment Model," Mathematical Finance, Vol. 10, No. 2, 2000, pp. 197-213. doi:10.1111/1467-9965.00089

[5] W. H. Fleming and S. J. Sheu, "Risk-Sensitive Control and an Optimal Investment Model. II,” Annals of Applied Probability, Vol. 12, No. 2, 2002, pp. 730-767. doi:10.1214/aoap/1026915623

[6] H. Hata, H. Nagai and S. J. Sheu, “Asymptotics of the Probability Minimizing a 'Down-Side' Risk,” Annals of Applied Probability, Vol. 20, No. 1, 2010, pp. 52-89. doi:10.1214/09-AAP618

[7] K. Kuroda and H. Nagai, "Risk Sensitive Portfolio Optimization on Infinite Time Horizon," Stochastics and Stochastics Reports, Vol. 73, No. 3-4, 2002, pp. 309-331.

[8] M. F. Bru, "Wishart Processes," Journal of Theoretical Probability, Vol. 4, No. 4, 1991, pp. 725-751. doi:10.1007/BF01259552

[9] C. Cuchiero, D. Filipovic, E. Mayerhofer and J. Teichmann, "Affine Processes on Positive Semidefinite Matrices," Annals of Applied Probability, Vol. 21, No. 2, 2011, pp. 397-463. doi:10.1214/10-AAP710

[10] E. Mayerhofer, O. Pfaffel and R. Stelzer, "On Strong Solutions for Positive Definite Jump Diffusions," Stochastic Processes and Their Applications, Vol. 121, No. 9, 2011, pp. 2072-2086. doi:10.1016/j.spa.2011.05.006

[11] A. Benabid, H. Bensusan and N. El Karoui, "Wishart Stochastic Volatility: Asymptotic Smile and Numerical Framework,” Preprint, 2010.
[12] J. Da Fonseca, M. Grasselli and C. Tebaldi, “Option Pricing When Correlations Are Stochastic: An Analytical Framework," Review of Derivatives Research, Vol. 10, No. 2, 2007, pp. 151-180. doi:10.1016/j.spa.2011.05.006

[13] J. Da Fonseca, M. Grasselli and C. Tebaldi, "A Multifactor Volatility Heston Model,” Quantitative Finance, Vol. 8, No. 6, 2008, pp. 591-604. doi:10.1080/14697680701668418

[14] C. Gouriéroux, "Continuous Time Wishart Process for Stochastic Risk,” Econometric Reviews, Vol. 25, No. 2, 2006, pp. 177-217.

[15] C. Gouriéroux, J. Jasiak and R. Sufana, "The Wishart Autoregressive Process of Multivariate Stochastic Volatility," Journal of Econometrics, Vol. 150, No. 2, 2009, pp. 167-181. doi:10.1016/j.jeconom.2008.12.016

[16] M. Grasselli and C. Tebaldi, "Solvable Affine Term Structure Models,” Mathematical Finance, Vol. 18, No. 1, 2008, pp. 135-153. doi:10.1111/j.1467-9965.2007.00325.x

[17] A. Buraschi, A. Cieslak and F. Trojani, "Correlation Risk and the Term Structure of Interest Rates,” Working Paper, University of St. Gallen, 2008.

[18] A. Buraschi, P. Porchia and F. Trojani, "Correlation Risk and Optimal Portfolio Choice,” Journal of Finance, Vol. 65, No. 1, 2010, pp. 393-420. doi:10.1111/j.1540-6261.2009.01533.x

[19] C. Chiarella, C.-Y. Hsiao and T.-D. Tô, "Risk Premia and Wishart Term Structure Models,” Preprint, 2010.

[20] C. Gouriéroux and R. Sufana, "Wishart Quadratic Term Structure Models,” Working Paper, CREF, 03-10, HEC, Montreal, 2003.

[21] D. Duffie, D. Filipovic and W. Schachermayer, "Affine Processes and Applications in Finance," Annals of Applied Probability, Vol. 13, No. 3, 2003, pp. 984-1053. doi:10.1214/aoap/1060202833

[22] D. Filipovic and E. Mayerhofer, "Affine Diffusion Processes: Theory and Applications," Radon Series on Computational and Applied Mathematics, Vol. 8, 2009, pp. 125-164. doi:10.1515/9783110213140.125

[23] H. Hata, "Down-Side Risk Probability Minimization Problem with Cox-Ingersoll-Ross's Interest Rates,” AsiaPacific Financial Markets, Vol. 18, No. 1, 2011, pp. 6987. doi:10.1007/s10690-010-9121-5

[24] Y. Watanabe, “Asymptotic Analyses for Certain Stochastic Control Problems under Partial Information and the Related Filtering Equation,” Thesis, Graduate School of Engineering Science, Osaka University, 2011.

[25] A. Bensoussan, "Stochastic Control of Partially Observable Systems,” Cambridge University Press, Cambridge, 1992. doi:10.1017/CBO9780511526503 